\title{
Effect of Regular Physical Activity and Lifestyle Changes on Insulin Resistance in Patients after Kidney Transplantation
}

\section{Graňák Karol}

Comenius University in Bratislava Jessenius Faculty of Medicine in Martin: Univerzita Komenskeho v

Bratislave Jesseniova Lekarska Fakulta v Martine

\section{Vnučák Matej}

Comenius University in Bratislava Jessenius Faculty of Medicine in Martin: Univerzita Komenskeho v Bratislave Jesseniova Lekarska Fakulta v Martine

\section{Skálová Petra}

Comenius University in Bratislava Jessenius Faculty of Medicine in Martin: Univerzita Komenskeho v Bratislave Jesseniova Lekarska Fakulta v Martine

\section{Pytliaková Margaréta}

Comenius University in Bratislava Jessenius Faculty of Medicine in Martin: Univerzita Komenskeho v Bratislave Jesseniova Lekarska Fakulta v Martine

\section{Laca Ludovít}

Comenius University in Bratislava Jessenius Faculty of Medicine in Martin: Univerzita Komenskeho v Bratislave Jesseniova Lekarska Fakulta v Martine

\section{Mokáň Marián}

Comenius University in Bratislava Jessenius Faculty of Medicine in Martin: Univerzita Komenskeho v Bratislave Jesseniova Lekarska Fakulta v Martine

Ivana Dedinská ( $\square$ idedinska@yahoo.co.uk)

Comenius University in Bratislava Jessenius Faculty of Medicine in Martin: Univerzita Komenskeho v Bratislave Jesseniova Lekarska Fakulta v Martine https://orcid.org/0000-0002-8246-4268

\section{Research}

Keywords: kidney transplantation, insulin resistance, physical activity

Posted Date: May 26th, 2021

DOl: https://doi.org/10.21203/rs.3.rs-549736/v1

License: (a) (i) This work is licensed under a Creative Commons Attribution 4.0 International License. Read Full License 


\section{Abstract}

Introduction: Insulin resistance (IR) at the time of kidney transplant (KT) is the most significant risk factor for the development of post-transplant diabetes mellitus (PTDM) and is a strong predictor of cardiovascular morbidity and mortality in patients after KT. It is possible to improve long-term survival of grafts and patients by influencing just modifiable risk factors, including obesity and the associated IR. The aim of this work is to determine the effect of regular physical activity and lifestyle changes on IR and other risk factors for PTDM in patients after KT.

Material and methods: The primary goal was to complete at least 150 minutes of moderate intensity physical exertion per week. Study group $(n=22)$ performed an aerobic or combined (aerobic + anaerobic) type of sports activity. Monitoring was provided by a sports tracker (Xiaomi Mi Band 4 compatible with Mi Fit mobile application). Control group was consisted of 22 stable patients after KT. Patients in both groups have the same immunosuppressive protocol. The duration of follow-up was 6 months.

Results: There were significantly lower waist circumference $(P=0.0437, P=0.0372)$, better graft function $(P=0.0036, P=0.0137)$, lower value of fasting blood glucose $(P=0.0016, P=0.0003), C$-peptide $(P=$ $0.0447, P=0014)$ in the 3rd and 6th month of monitoring and lower low-density lipoprotein at 6 months $(P=0.0444)$ in the observed group compared to the control group. IR was statistically significantly lower at 6 months $(P=0.0202)$ and fasting blood glucose at 3 and 6 months $(P=0.0227)$ in the observed group. In the monitored group, the subgroup performing intensive training achieved a significant additional effect on the reduction of waist circumference $(P=0.0173)$.

Conclusion: In our study, we confirmed a significant effect of regular physical activity in preventing the development of IR and impaired fasting glucose in patients after KT. It is necessary to perform at least 150 minutes of medium-intensity aerobic or combined sport effort per week to achieve this goal.

\section{Introduction}

Patients after kidney transplantation (KT) have a higher incidence of insulin resistance (IR) compared to the age and sex matched general population (1). IR is the most important predictor of post-transplant diabetes mellitus (PTDM), what was confirmed in a multicenter study on the population of KT recipients in the Slovak Republic (2). As with type 2 diabetes mellitus (DM), pathogenesis of PTDM has been associated with impaired insulin-mediated glucose uptake by peripheral tissues, as well as impaired insulin-mediated suppression of hepatic glucose release. In their study using a hyperinsulinemiceuglycemic clamp, the Danish authors found a decrease in insulin sensitivity 6 months after KT, which was characterized by impaired suppression of endogenous glucose production and excretion in the liver and whole-body lipolysis (3). However, IR itself is not manifested by hyperglycemia as long as B-cells are able to compensate and therefore their dysfunction is probably necessary to bridge to PTDM (4).

Insulin resistance plays an important role in the development of cardiovascular diseases (5). It is cardiovascular morbidity and mortality that significantly limit the long-term survival of patients after KT. 
The incidence and prevalence of cardiovascular diseases is 5 times higher in this group than in the general population (6). It even appears that patients with impaired glucose tolerance in the posttransplant period have the same risk of mortality as patients with already confirmed PTDM (7). The main risk factors for IR after KT are weight gain and related obesity, low physical activity and chronic use of immunosuppressive therapy. Oterdoom et al. confirmed in their study, that obesity, its distribution, and prednisolone dose are fundamental determinants of IR in the long term after CT (6). Intense lifestyle changes, decreased caloric intake, weight reduction, and exercise are well-established steps to prevent pre-diabetic conditions and type $2 \mathrm{DM}$ with a long-lasting effect in non-transplant population $(8,9)$. It is the active influence of modifiable risk factors that may be the key to preventing the development of IR after KT.

Being physically active is one of the most important steps a person of all ages can work to improve their health. A sedentary lifestyle can affect patients at all stages of chronic kidney disease (CKD), including patients after KT (10). Low physical activity has been identified as a major modifiable risk factor for mortality in patients with end-stage CKD (11). KT offers these patients the elimination of factors that contribute to a sedentary lifestyle, such as time spent on dialysis, anemia, uremia, or reduced production of certain hormones (12). The effect of insulin in the liver and muscles can be modified by regular physical exertion. Regular training increases muscle capillary density, oxidative capacity, lipid metabolism and supports insulin signaling pathways. If the primary goal is to improve the effect of insulin, daily moderate or high intensity activity is optimal. The most significant improvement was observed in those who had the highest baseline IR (13).

The 2009 KDIGO (Kidney Disease: Improving Global Outcome) recommendations for the management of patients after KT contains only vague guidelines where these patients are strongly encouraged to follow a healthy lifestyle through exercise, proper diet, and weight reduction as needed (14). There are few studies addressing this issue, based on subjective self-assessment questionnaires and usually uncontrolled. The aim of our study is to determine the effect of regular physical activity in the prevention of IR and other risk factors (hyperlipidemia, fasting hyperglycemia, weight gain) for PTDM in patients after KT. The secondary goal is to evaluate its influence on the development of graft function. The specificity is objectively monitored sports activity using digital technologies.

\section{Material And Methods}

This is a prospective controlled analysis. The study group consisted of patients $(n=44)$ after primary living-donor or deceased-donor KT at the Martin Transplant Center. The total length of follow-up was 6 months and all patients undergoing 3 examinations during the follow-up period: admission, at 3 months, and at 6 months.

Collaborating patients at least 3 months after KT with good stable graft function, as defined by estimated glomerular filtration rate (eGFR) $\geq 60 \mathrm{ml} / \mathrm{min} / 1.73 \mathrm{~m} 2$ according to the Chronic Kidney Disease Epidemiology Collaboration Index (CKD-EPI), were included in the cohort. Exclusion criteria were: 
diagnosed with DM, PTDM and pre-diabetic conditions (fasting hyperglycemia, impaired glucose tolerance), age over 65 years and hemoglobin value $<100 \mathrm{~g} / \mathrm{l}$. All patients received the same combination of immunosuppressive drugs in a maintenance regimen (tacrolimus, mycophenolic acid, prednisone). We divided the file into two groups.

All patients completed an initial interview prior to enrollment in the study. The interview focused on their life style, diet routine and physical acitivity. Patients with regular physical acitivity before were excluded from the study.

The intervention group consisted of 22 patients for whom a limit of at least 150 minutes of moderate exercise per week was set. This limit is based on the 2016 recommendations of the American Diabetes Associaton (ADA) for the prevention and treatment of DM and pre-diabetic conditions in the general population (15). At the same time, patients were advised not to have a break of more than 2 days between exercises. The permitted type of activity was aerobic (running, swimming, brisk walking, cycling) or combined (aerobic + strength) physical activity, according to the patient's preferences. Monitoring of physical activity was provided using a Xiaomi Mi Band4 sports bracelet compatible with the Mi Fit mobile application, where the characteristics of the performed activity for the entire monitored period were stored (duration and frequency per week, total energy expenditure, type of sports activity, heart rate). The intensity of physical activity was assessed using the \% of maximum heart rate (HRmax), which is determined using a simple scheme: 220 - patient age. The level of physical activity of medium intensity represents $64-76 \%$ of HRmax and of high intensity 77-93\% (16). A control group $(n=22)$ was created for the intervention group, whose members met all the inclusion and exclusion criteria of the study. These patients were instructed on routine measures while maintaining a stable body weight and waist circumference through active lifestyle and dietary measures. However, compared to group 1, they were not prescribed a minimum level of physical activity and were not monitored during follow-up. At the end of the study they completed a questionnaire describing their life style. None of the patients in the control group met the criteria for the intervention group exercise limit per week during the monitored period.

The parameters recorded for each patient are listed in Table 1. Laboratory and anthropometric parameters were monitored at each outpatient check-up. To evaluate IR, we used the HOMA-IR (Homeostatic Model Assessment of Insulin Resistance) index, which is calculated by multiplying fasting plasma glucose (FPG) and fasting insulin (FPI) values divided by 22.5, and thus HOMA-IR = (FPG $\times$ FPI) / 22.5. Proteinuria was examined from 24-hour urine collection. Input parameters included a history of cytomegalovirus (CMV) replication (cut-off was $1000 \mathrm{cop} / \mathrm{ml}$ ) requiring transient reduction or discontinuation of mycophenolic acid and 21-day valganciclovir treatment. All patients had stable fasting tacrolimus levels maintained in the recommended range of 3.0-6.0 ng/L during the follow-up period. The daily dose of prednisone in both groups was also recorded regularly. A positive family history for DM means its confirmed occurrence in siblings, parents and grandparents.

Table 1. Examination protocol 


\begin{tabular}{|c|c|c|c|}
\hline Monitored parameters & Base line & 3. month & 6. month \\
\hline Age (years) & $\mathrm{x}$ & & \\
\hline Body weight (kg) & $\mathrm{x}$ & $\mathrm{x}$ & $\mathrm{x}$ \\
\hline BMI $\left(\mathrm{kg} / \mathrm{m}^{2}\right)$ & $x$ & $\mathrm{x}$ & $\mathrm{x}$ \\
\hline Waist circumference $(\mathrm{cm})$ & $x$ & $\mathrm{x}$ & $\mathrm{x}$ \\
\hline DM positive family history (yes/no) ${ }^{2}$ & $\mathrm{x}$ & & \\
\hline Cause of renal failure & $\mathrm{x}$ & & \\
\hline Time after $\mathrm{KT}(\mathrm{M})$ & $x$ & & \\
\hline Induction immunosuppression & $\mathrm{x}$ & & \\
\hline \multicolumn{4}{|l|}{ (thymoglobulin/basiliximab) } \\
\hline Smoking (yes/no) & $\mathrm{x}$ & & \\
\hline Anamnesis of acute rejection (yes/no, type) ${ }^{b}$ & $x$ & & \\
\hline Anamnesis of CMV & $\mathrm{x}$ & & \\
\hline Delayed graft function (yes/no) ${ }^{c}$ & $\mathrm{x}$ & & \\
\hline Serum magnesium (mmol/l) & $\mathrm{x}$ & $\mathrm{x}$ & $\mathrm{x}$ \\
\hline Creatinine (umol/l) & $x$ & $\mathrm{x}$ & $x$ \\
\hline eGFR CKD-EPI $\left(\mathrm{ml} / \mathrm{min} / 1.73 \mathrm{~m}^{2}\right)$ & $x$ & $\mathrm{x}$ & $x$ \\
\hline Quantitative proteinuria $(\mathrm{g} / \mathrm{l})$ & $x$ & $\mathrm{x}$ & $\mathrm{x}$ \\
\hline Hemoglobin $(\mathrm{g} / \mathrm{l})$ & $x$ & $\mathrm{x}$ & $x$ \\
\hline Vitamin D (ug/l) & $x$ & $\mathrm{x}$ & $x$ \\
\hline Fasting glucose $(\mathrm{mmol} / \mathrm{l})$ & $x$ & $\mathrm{x}$ & $x$ \\
\hline Immunoreactive protein - IRI (ulU/ml) & $x$ & $\mathrm{x}$ & $x$ \\
\hline C-peptide (pmol/l) & $x$ & $\mathrm{x}$ & $x$ \\
\hline Glycated hemoglobin - $\mathrm{HbA1}_{\mathrm{c}}(\%)$ & $\mathrm{x}$ & $\mathrm{x}$ & $x$ \\
\hline HOMA-IR & $\mathrm{x}$ & $\mathrm{x}$ & $x$ \\
\hline TAG - Triglycerides (mmol/l) & $\mathrm{x}$ & $\mathrm{x}$ & $x$ \\
\hline Cholesterol (mmol/l) & $x$ & $\mathrm{x}$ & $x$ \\
\hline LDL - low-density lipoprotein (mmol/l) & $x$ & $\mathrm{x}$ & $\mathrm{x}$ \\
\hline HDL - high-density lipoprotein (mmol/l) & $x$ & $\mathrm{x}$ & $\mathrm{x}$ \\
\hline
\end{tabular}

BMI - body mass index; KT - kidney transplant; CMV - cytomegalovirus; eGFR - estimated glomerular filtration rate; CKD-EPI - Chronic Kidney Disease

Epidemiology Collaboration; HOMA-IR - homeostatic model assessment for insulin resistance.

a Siblings, parents, grandparents.

b Acute cellular rejection treated methylprednisolone or acute combined rejection treated methylprednisolone + plasmapheresis / Intravenous Immunoglobulins / rituximab.

c Need for dialysis within 7 days after KT.

Finally, we divided the monitored group into 4 subgroups according to the level of physical activity (duration, intensity): 1 . met only the primary endpoint ( $\geq 150$ minutes and medium intensity), 2 . $\geq 150$ minutes and high intensity, 3 . $\geq 300$ minutes and medium intensity 4 . $\geq 300$ minutes and high intensity. According to the type of physical activity, we also divided it into 2 subgroups: a) aerobic activity, b) 
combined activity. We compared HOMA-IR as well as other monitored characteristics between individual subgroups.

We used a certified statistical program, MedCalc version 13.1.2. (MedCalc Software VAT registration number BE 0809 344,640, Member of International Association of Statistical Computing, Ostend, Belgium). Comparisons of continuous variables between groups were carried out using parametric (t-test) or non-parametric (Mann-Whitney) tests; associations between categorical variables were analyzed using the $\chi^{2}$ test and Fisher's exact test, as appropriate. P-value $<0.05$ was considered to be statistically significant.

\section{Results}

A total of 44 patients were enrolled in the study, of whom 22 were the monitoring group and 22 were the control group. Table 2 shows the basic characteristics of the group. There was no significant difference between the two groups in terms of gender $(P=0.7677)$ and age structure $(P=0.9531)$. Patients in the control group had a significantly shorter time from KT compared to the monitored group $(P=0.0002)$, but the mean time interval from KT in this group was $15.8 \pm 9$ months, and thus the inclusion criterion was met (minimum 3 months after KT). There was no difference between the two groups in the daily dose of prednisone or in the use of basiliximab in the induction protocol. In Fig. 1, we can see the composition of the group according to the main cause of kidney failure (Fig. 1). The largest part was chronic glomerulonephritis and chronic tubulointerstitial nephritis. Of the 22 patients in the monitored group, 15 performed isolated aerobic activity and 7 combined (aerobic + strengthening). The types of sports practiced were running, cycling, brisk walking, hiking, swimming and weight or bodyweight training. 
Table 2

Group characteristics

Group characteristics

monitored group control group P-value

$\mathrm{n}=\mathbf{2 2}$

$\mathrm{n}=\mathbf{2 2}$

Basic group characteristics

Gender - men (\%)

Age (years)

Time after KT (M)

Basiliximab in induction (\%)

Delayed graft function (\%)

DM positive family history (\%)

Smokers (\%)

Anamnesis of $\mathrm{CMV}(\%)$

Anamnesis of acute rejection

Average prednisone dose (mg/day)

\section{0}

$42,6 \pm 8,8$

$60,6 \pm 50$

36,4

4,5

41

9

13,6

18

$5,9 \pm 2,4$

Anthropometric data

Body weight $(\mathrm{kg})$ base line

Body weight $(\mathrm{kg}) 3 \mathrm{M}$

Body weight $(\mathrm{kg}) 6 \mathrm{M}$

BMI $(\mathrm{kg} / \mathrm{m} 2)$ base line

BMI $(\mathrm{kg} / \mathrm{m} 2) 3 \mathrm{M}$

BMI $(\mathrm{kg} / \mathrm{m} 2) 6 \mathrm{M}$

Waist circumference $(\mathrm{cm})$ base line

Waist circumference (cm) 3M

Waist circumference $(\mathrm{cm}) 6 \mathrm{M}$

Body height (cm)

Creatinine $(\mu \mathrm{mol} / \mathrm{l})$ base line

KT - kidney transplant; DM - diabetes mellitus; CMV - cytomegalovirus; BMI - body mass index; $\mathrm{M}$ - month; eGFR - estimated glumerular filtration rate; CKD-EPI - Chronic Kidney Disease

Epidemiology Collaboration; HOMA-IR - homeostatic model assessment for insulin resistance.

\begin{tabular}{lcl}
\hline $75 \pm 14,3$ & $77 \pm 16,4$ & 0,6686 \\
\hline $74,9 \pm 13,8$ & $78,3 \pm 16,4$ & 0,4610 \\
\hline $75,1 \pm 13,4$ & $79,7 \pm 17$ & 0,3246 \\
\hline $25,5 \pm 3,2$ & $25,5 \pm 3,8$ & 1,0000 \\
\hline $25,4 \pm 3$ & $26 \pm 3,7$ & 0,5578 \\
\hline $25,5 \pm 2,9$ & $26,4 \pm 4$ & 0,3977 \\
\hline $90,6 \pm 12,4$ & $94,1 \pm 12,2$ & 0,3507 \\
\hline $89,3 \pm 11,5$ & $96,7 \pm 12,1$ & 0,0437 \\
\hline $89,1 \pm 11,1$ & $96,7 \pm 12,3$ & 0,0372 \\
\hline $171 \pm 8,2$ & $173 \pm 11,7$ & 0,5150 \\
\hline Laboratory parameters - graft function \\
\hline 95,1 $\pm 17,4$ & $101,4 \pm 23,3$ & 0,3154
\end{tabular}




\begin{tabular}{|c|c|c|c|}
\hline \multirow[t]{2}{*}{ Group characteristics } & monitored group & control group & P-value \\
\hline & $\mathrm{n}=\mathbf{2 2}$ & \multicolumn{2}{|l|}{$\mathrm{n}=22$} \\
\hline Creatinine $(\mu \mathrm{mol} / \mathrm{l}) 3 \mathrm{M}$ & $98,2 \pm 19,2$ & $114,5 \pm 30,1$ & 0,0381 \\
\hline Creatinine $(\mu \mathrm{mol} / \mathrm{l}) 6 \mathrm{M}$ & $94,5 \pm 22,1$ & $110,1 \pm 27,1$ & 0,0425 \\
\hline eGFR CKD-EPI (ml/min) base line & $76,4 \pm 15,5$ & $72,7 \pm 18,9$ & 0,4816 \\
\hline eGFR CKD-EPI (ml/min) 3M & $74,1 \pm 16$ & $63,4 \pm 16,3$ & 0,0036 \\
\hline eGFR CKD-EPI (ml/min) 6M & $78,2 \pm 17,5$ & $65,7 \pm 14,6$ & 0,0137 \\
\hline Quantitative proteinuria $(\mathrm{g} / \mathrm{l})$ base line & $0,236 \pm 0,15$ & $0,220 \pm 0,18$ & 0,7503 \\
\hline Quantitative proteinuria $(\mathrm{g} / \mathrm{l}) 3 \mathrm{M}$ & $0,220 \pm 0,19$ & $0,267 \pm 0,29$ & 0,5383 \\
\hline Quantitative proteinuria $(\mathrm{g} / \mathrm{l}) 6 \mathrm{M}$ & $0,187 \pm 0,13$ & $0,347 \pm 0,42$ & 0,0952 \\
\hline Vitamin $D(\mu g / l)$ base line & $31,9 \pm 10,2$ & $23,2 \pm 7,2$ & 0,0022 \\
\hline Vitamin D $(\mu \mathrm{g} / \mathrm{l}) 3 \mathrm{M}$ & $29,5 \pm 8,9$ & $24 \pm 8$ & 0,0369 \\
\hline Vitamin D $(\mu \mathrm{g} / \mathrm{l}) 6 \mathrm{M}$ & $27,9 \pm 8,8$ & $26,5 \pm 10$ & 0,6246 \\
\hline Hemoglobin $(g / l)$ base line & $143 \pm 12,1$ & $133 \pm 15$ & 0,0193 \\
\hline Hemoglobin $(g / l) 3 M$ & $145 \pm 10,3$ & $137 \pm 16,5$ & 0,0605 \\
\hline \multirow[t]{2}{*}{ Hemoglobin $(g / l) 6 M$} & $146 \pm 11,5$ & $142 \pm 16,7$ & 0,3601 \\
\hline & \multicolumn{3}{|c|}{ Laboratory parameters - glucose metabolism } \\
\hline Fasting glucose $(\mathrm{mmol} / \mathrm{l})$ base line & $4,6 \pm 0,5$ & $5,2 \pm 0,5$ & 0,0003 \\
\hline Fasting glucose $(\mathrm{mmol} / \mathrm{l}) 3 \mathrm{M}$ & $4,8 \pm 0,6$ & $5,7 \pm 1,1$ & 0,0016 \\
\hline Fasting glucose $(\mathrm{mmol} / \mathrm{l}) 6 \mathrm{M}$ & $4,8 \pm 0,6$ & $5,7 \pm 0,9$ & 0,0003 \\
\hline C - peptide $(\mu \mathrm{g} / \mathrm{l})$ base line & $2,5 \pm 1$ & $3,1 \pm 1,2$ & 0,0788 \\
\hline C - peptide $(\mu g / l) 3 M$ & $2,2 \pm 0,8$ & $2,8 \pm 1,1$ & 0,0447 \\
\hline C - peptide $(\mu g / l) 6 \mathrm{M}$ & $2,4 \pm 0,9$ & $4 \pm 2$ & 0,0014 \\
\hline Immunoreactive insulin - IRI (mU/l) base line & $8,4 \pm 6,2$ & $7,8 \pm 3,2$ & 0,6887 \\
\hline Immunoreactive insulin - IRI (mU/I) 3M & $8,3 \pm 6,8$ & $7,9 \pm 3,4$ & 0,8063 \\
\hline Immunoreactive insulin - IRI (mU/l) 6M & $8,7 \pm 4,7$ & $9,7 \pm 3,7$ & 0,4374 \\
\hline HOMA-IR base line & $1,7 \pm 1,3$ & $1,8 \pm 0,8$ & 0,7601 \\
\hline
\end{tabular}

KT - kidney transplant; DM - diabetes mellitus; CMV - cytomegalovirus; BMI - body mass index; $\mathrm{M}$ - month; eGFR - estimated glumerular filtration rate; CKD-EPI - Chronic Kidney Disease

Epidemiology Collaboration; HOMA-IR - homeostatic model assessment for insulin resistance. 


\begin{tabular}{|llll|}
\hline Group characteristics & monitored group & control group & P-value \\
\hline HOMA-IR 3M & $\mathbf{n = 2 2}$ & $\mathbf{n = 2 2}$ & \\
\hline HOMA-IR 6M & $1,8 \pm 1,5$ & $2,4 \pm 1,2$ & 0,1504 \\
\hline Glycated hemoglobin - HbA1c (\%) base line & $1,9 \pm 1$ & $2,5 \pm 1,1$ & 0,0653 \\
\hline Glycated hemoglobin - HbA1c (\%) 3M & $3,6 \pm 0,4$ & $3,8 \pm 0,7$ & 0,2512 \\
\hline Glycated hemoglobin - HbA1c (\%) 6M & $3,6 \pm 0,5$ & $3,6 \pm 0,5$ & 1,0000 \\
\hline & $3,6 \pm 0,5$ & $3,9 \pm 0,7$ & 0,1094 \\
\hline Cholesterol (mmol/I) base line & Laboratory parameters - lipid profile & \\
\hline Cholesterol (mmol/I) 3M & $5 \pm 0,8$ & $5,3 \pm 1$ & 0,2781 \\
\hline Cholesterol (mmol/I) 6M & $4,9 \pm 0,8$ & $5,1 \pm 1$ & 0,4679 \\
\hline LDL - low-density lipoprotein (mmol/I) base line & $2,8 \pm 0,7$ & $5,2 \pm 1$ & 0,1504 \\
\hline LDL - low-density lipoprotein (mmol/I) 3M & $2,8 \pm 0,7$ & $3,2 \pm 0,8$ & 0,0848 \\
\hline LDL - low-density lipoprotein (mmol/I) 6M & $2,7 \pm 0,8$ & $3 \pm 0,9$ & 0,4153 \\
\hline HDL - high-density lipoprotein (mmol/I) base line & $1,5 \pm 0,4$ & $3,2 \pm 0,8$ & 0,0444 \\
\hline HDL - high-density lipoprotein (mmol/I) 3M & $1,5 \pm 0,5$ & $1,3 \pm 0,4$ & 0,1047 \\
\hline HDL - high-density lipoprotein (mmol/I) 6M & $1,5 \pm 0,5$ & $1,4 \pm 0,5$ & 0,5107 \\
\hline TAG - triglycerides (mmol/I) base line & $2 \pm 1,4$ & $1,4 \pm 0,4$ & 0,4679 \\
\hline TAG - triglycerides (mmol/I) 3M & $1,5 \pm 0,9$ & $2,2 \pm 1$ & 0,5885 \\
\hline TAG - triglycerides (mmol/I) 6M & $1,7 \pm 0,8$ & $1,8 \pm 0,7$ & 0,2240 \\
\hline KT - kidney transplant; DM - diabetes mellitus; CMV - cytomegalovirus; BMI - body mass index; M & 0,2492 \\
\hline Epidemiology Collaboration; HOMA-IR - homeostatic model assessment for insulin resistance. \\
\hline
\end{tabular}

By monitoring anthropometric parameters, we found a statistically significant decrease in waist circumference in the monitored group at $3(P=0.0437)$ and 6 months $(P=0.0372)$ after KT compared to the control group. On the contrary, we did not observe a significant difference in the development of body weight and $\mathrm{BMI}$ between the two groups.

Patients in the control group had a significant increase in creatinine compared to the intervention group at $3(P=0.0381), 6$ months $(P=0.0425)$ and at the same time showed poorer graft function expressed by eGFR according to CKD-EPI $(P=0.0036), P=(0.0137)$. At the beginning of the follow-up they suffered from significantly lower levels of vitamin $\mathrm{D}$. This trend continued in the 3rd month of the follow-up, but 
had an increasing tendency, on the contrary decreasing in the monitored group, and at the end of the follow-up we did not observe a significant difference in its level. The hemoglobin level was initially lower in the control group, but on average it was in the zone of very mild anemia and thus met the inclusion criterion. At the same time, it was saturated during follow-up and the differences between the groups leveled off.

We also noted differences in glucose and lipid metabolism. In the intervention group, fasting plasma glucose was significantly lower at baseline $(P=0.0045)$, at $3(P=0.0016)$, and at 6 months $(P=0.0003)$. However, higher baseline glycemia in the control group falls within the normoglycemic range and does not represent a pathological condition. Also, in the intervention group, we found a significantly lower value of $C$-peptide at $3(P=0.0447)$ and 6 months $(P=0.0014)$. The lipid profile indicated significantly lower LDL cholesterol in this group after the end of follow-up $(P=0.0444)$.

We confirmed that there was a significant increase in HOMA-IR in the control group after the end of follow-up ( $P=0.0202)$ (Fig. 2). Fasting blood glucose increased significantly in this group as early as month 3 , and a significant difference persisted at the end of follow-up $(P=0.0227)$ (Fig. 3).

In the monitored group, according to the level of physical activity, 7 patients (1st subgroup) met the primary endpoint only, 5 patients met the conditions of the 2nd subgroup, 6 patients of the 3rd subgroup and 4 patients met the 4 th subgroup. No significant differences in HOMA-IR and other parameters of glucose and fat metabolism were found between the subgroups. Subgroup 4 with the highest level of physical exertion showed a significant reduction in waist circumference at the end of follow-up ( $P=$ 0.0173) (Fig. 4). We also did not notice a difference in IR by comparing subgroups with isolated aerobic $(n=15)$ and combined $(n=7)$ training. Patients practicing only aerobic type activity achieved a significant decrease in triglycerides after the end of follow-up $(P=0.046)($ Fig. 5$)$.

\section{Discussion}

Insulin resistance is a strong risk factor for the development of PTDM and diseases of the cardiovascular system, which significantly affect the long-term survival of patients after KT. Modifiable factors, such as weight gain in the absence of regular physical activity, contribute significantly to these insulin-resistant patients much more frequently compared to their peers in the general population (6).

It is known and verified by several studies that regular physical activity is an effective tool for the prevention and treatment of pre-diabetic conditions and type $2 \mathrm{DM}$, which clearly strengthens its position among interventional procedures in this group of patients $(17,18)$. Conversely, in the transplanted population, the data are severely limited. Sharif et al. in 2008 they were the first to monitor the effect of intensive lifestyle modification in patients after KT. Physical activity included 2 hours of endurance discipline per week. However, the study was designed primarily for therapeutic purposes, as subjects had already been diagnosed with IGT or PTDM. At 6-month follow-up, there was a 15\% improvement in 2-hour postprandial glycemia versus $12 \%$ deterioration in the control group. In the first group, $44 \%$ of those treated with IGT developed normal glucose tolerance, while only $4 \%$ switched to PTDM and $58 \%$ of those 
monitored with PTDM achieved improvement (29\% to IGT, $29 \%$ to normal tolerance). The study pointed out the importance and significance of active intervention in the lifestyle of these patients, both in the prevention of high-risk patients and in the treatment of already developed PTDM. However, the evaluation of sporting activity was subjective (19).

Recently, a prospective randomized study CAVIAR (Comparing Glycemic Benefits of Active Versus Passive Lifestyle Intervention in Kidney Allograft Recipients) was published, comparing the effect of a complex lifestyle change on glucose metabolism among patients after KT without PTDM. 130 recipients were randomized 1:1 into active (lifestyle counseling by a renal dietitian using a behavior change technique) and passive intervention groups. As in our study, the length of follow-up was 6 months. In terms of physical activity, patients were encouraged to increase their performance and kept a training diary. This study did not confirm a different impact of active versus passive intervention on insulin sensitivity or secretion (20). The Dutch multicenter randomized study ACT (The Active Care after Transplantation) is currently underway, comparing 3 groups of patients (classic care, physical exercise intervention and combination of diet and exercise) with the primary aim to assess the impact of lifestyle changes on quality of life as well as degree of physical condition, adipose tissue and cardio-metabolic risk factors such as blood pressure, lipids, glucose metabolism (21).

Weight gain and obesity are known risk factors for the development of IR in the post-transplant period. Oterdoom et al. in their work they state that obesity evaluated by the BMI index, but also the distribution of adipose tissue determined from the waist-to-hip ratio are the strongest determinants for the development of IR after KT. Even adipose tissue distribution is a risk factor independent of general obesity, and therefore assessment of waist circumference provides more accurate information on abdominal obesity than BMI (6). In our study, regular physical activity led to a significant reduction in waist circumference and a reduction in LDL cholesterol compared to the control group. However, the reduction in body weight, $\mathrm{BMI}$, waist circumference and lipid profile parameters did not reach statistically significant in the multivariate model. In the CAVIAR study, the active intervention group achieved significant progress in weight and fat mass reduction in contrast to the passive group, which clearly contributes to improving the cardio-metabolic risk profile of these patients (20). We also demonstrated a significant difference in waist circumference value reduction between patients in the intervention group, which was achieved by the subgroup with the highest level of sports exertion, which supports a direct relationship between the degree of physical activity and cardio-metabolic risk. In 2011, Zelle et al. in a prospective study on a sample of 540 recipients after KT, they confirmed a strong independent correlation between low physical activity and cardiovascular and overall mortality (22). Recently, Byambasukh et al. published work in which recipients performing moderate to high intensity physical activity showed significantly lower cardiovascular and overall mortality compared to recipients with a sedentary lifestyle regardless of age, gender, and graft function (23).

The secondary goal of our work was to evaluate the influence of physical activity on the development of graft function. We found a significantly lower value of serum creatinine and a higher eGFR already in the 3rd month from the beginning of the follow-up in the intervention group in comparison with the control 
group. We assume that the length of our follow-up is relatively short in terms of graft function impact assessment. The Italian authors Totti et al. reported a significant increase in creatinine and a decrease in eGFR during the 3-year follow-up period in the passive group compared to patients exercising regularly $\geq$ 150 minutes per week (24).

In our study, we confirmed that regular exercise of moderate intensity of at least 150 minutes per week is sufficient to adequately prevent glucose metabolism disorders. This conclusion is in line with the ADA recommendations for high-risk patients or with prediabetes to prevent and delay the development of DM (15). The WHO (World Health Organization) also proposes the same minimum weekly sports activity limit in its 2010 recommendations for the adult population. At the same time, they state that in order to achieve an additional health effect, it is necessary to increase this activity to $\geq 300$ minutes of medium intensity per week or $\geq 150$ minutes of high intensity per week (25). In our study, such an effect (significant reduction in waist circumference) was achieved by a subgroup meeting the criteria of highintensity and long-lasting exertion ( $\geq 300$ minutes per week). In most available works, an aerobic type of sport is applied to evaluate the effect of physical activity. Both aerobic and strength training are known to promote skeletal muscle, adipose tissue and liver adaptation in association with increased insulin action (13). The combination of endurance and strength training can provide even greater improvement and appears to be superior to continuous aerobic training (26). The ADA and WHO also recommend incorporating a strength component into regular training $(15,25)$. By comparing the combined activity with aerobic, we did not find in our study an additional impact on the parameters of glucose metabolism or anthropometric data. At the end of the follow-up, the group with isolated aerobic activity showed a significantly lower value of triglycerides.

There are several similarities between glucose metabolism disorders in the general population and arising de novo after KT, but the transplanted population is exceptional in many aspects and needs to be approached in an individualized way. Therefore, there are no specific suggestions for prevention, but also non-pharmacological treatment of these disorders in the general recommendations for recipient care after $\mathrm{KT}$, especially at a time when the KT patient is not rare in the population and a healthy lifestyle is the number one topic in most age groups. Based on the findings, we consider it necessary to apply regular physical activity, but also dietary counseling to daily practice as much as possible in order to improve the long-term survival of grafts and patients after KT.

We consider the incorporation of digital technologies for patient monitoring and thus the simple verification of data, which in our case is not based only on the questionnaire method, to be an important and unique aspect of this study. Sports bracelets, which in most cases are already part of the watch, are now widely available and commonly used from beginners to professional athletes of various ages. They use motion and thermally controlled sensors to record energy expenditure and monitor metabolic physical activity, thus providing us with an objective evaluation of each patient's athletic performance. By pairing them with a mobile application, we obtained a detailed analysis of the entire period under review, and on the other hand, we consider them to be an important factor in motivating individuals and striving to improve their results. To date, no studies have performed on the transplanted sample of patients to 
objectively evaluate the impact of physical activity on changes in glucose metabolism. We consider this an excellent opportunity to further monitor the impact of lifestyle modifications on the development of the cardio-metabolic profile of this risk group of patients. The possible different impact of lifestyle adjustments depending on gender remains questionable, given that in a study in 2019 by Dedinská et al. found that in men after KT, IR and metabolic syndrome are the main predictors of PTDM, while in women it is pancreatic $\beta$ cell dysfunction (27). It is therefore necessary to incorporate this probably important variable into further research.

The limitation of the study is the small number of enrolled patients and the possible problem with monitoring physical activity carried out in the control group. There was significantly different time from kidney transplantation between both groups with worse kidney function however there were no differences in immunosuppression and average kidney function was excellent in both groups.

\section{Conclusion}

Performing regular physical activity leads to a significant reduction in the development of IR in patients after KT. To achieve this goal in our study, it was necessary to complete at least 150 minutes of physical effort of medium intensity per week. An isolated aerobic and combined (aerobic + strength) type of sport seems to be equally effective.

Patients after KT are at higher risk of developing IGT and PTDM, which is also reflected in its high incidence in the given population. Therefore, in each transplanted patient, it is necessary to examine the parameters of glucose metabolism, including the oral glucose tolerance test (OGTT) with the initiation of aggressive lifestyle modification in those with a detected pre-diabetic condition or PTDM. However, it is extremely important that patients with normal glucose tolerance also received enough attention, and that the emphasis on lifestyle (especially regular physical activity) is a cornerstone of prevention, as in the general population.

\section{Abbreviations}




\begin{tabular}{|ll|}
\hline ADA & American Diabetes Association \\
\hline ADPKD & autosomal dominant polycystic kidney disease \\
\hline BMI & body mass index \\
\hline CKD & chronic kidney disease \\
\hline CKD-EPI & Chronic kidney disease - Epidemiology Collaboration Index \\
\hline CMV & cytomegalovirus \\
\hline DGF & delayed graft function \\
\hline DM & diabetes mellitus \\
\hline eGFR & estimated glomerular filtration rate \\
\hline FPG & fasting plasma glucose \\
\hline FPI & fasting plasma insulin \\
\hline GNF & glomerulonephritis \\
\hline HbA1 & glycated hemoglobin \\
\hline HDL & high-density lipoprotein \\
\hline HOMA-IR & Homeostatic Model Assesment of Insulin Resistance \\
\hline HRmax & maximum heart rate \\
\hline IR & insulin resistance \\
\hline IRI & immunoreactive insulin \\
\hline KDIGO & Kidney disease: Improving Global Outcome \\
\hline KT & kidney transplant \\
\hline LDL & low-density lipoprotein \\
\hline M & month \\
\hline OGTT & oral glucose tolerance test \\
\hline PTDM & post-transplant diabetes mellitus \\
\hline TAG & triglycerides \\
\hline TIN & tubulointerstitial nephritis \\
\hline WHO & World Health Organization \\
\hline
\end{tabular}




\section{Declarations}

\section{Ethical approval and consent to participate:}

All procedures involving human participants have been approved according to the ethical standards of the institutional research committee, including the 1964 Helsinki Declaration and its later amendments of comparable ethical standards.

The clinical and research activities being reported are consistent with the Principles of the Declaration of Istanbul as outlined in the Declaration of Istanbul on Organ Trafficking and Transplant Tourism. No organs were obtained from prisoners and organs were obtained via National - Slovak transplant organization.

Informed consent was obtained from all individual participants included in the study.

Consent for publication: Patients signed informed consent regarding publishing their data.

\section{Availability of data and material:}

Informed consent for included participants was checked and approved by University hospital's ethical committee and all signed informed consents have been archived for at least 20 years after research was completed.

Competing interests: The authors declare no conflicts of interest.

Funding: None

\section{Authors' contributions:}

Karol Graňák, MD: participated in performing the research and data analysis and writing the paper

Matej Vnučák, MD, Ph.D: participated in data collection

Petra Skalová, MD: participated in data collection

Margaréta Pytliaková, MD: participated in performing the research

Prof. L'udovít Laca, MD, Ph.D: participated in writing the paper

Prof. Marián Mokáň, MD, DrSc, FRCP Edin: participated in research design

Assoc. prof. Ivana Dedinská, MD, Ph.D: participated in performing the research and data analysis 


\section{References}

1. Hjelmesaeth $\mathrm{J}$, Midtvedt $\mathrm{K}$, Jenssen $\mathrm{T}$, et al. Insulin resistance after renal transplantation: impact of immunosuppressive and antihypertensive therapy. Diabetes Care. 2001;24:2121-6.

2. Dedinská I, Baltesová T, Beňa L, et al Incidence of Diabetes Mellitus After Kidney Transplantation in Slovakia: Multicentric, Prospective Analysis. Transplantation Proceedings, 48, 3292-3298 (2016).

3. Jorgensen MB, Hornum M, van Hall G, et al. The impact of kidney transplantation on insulin sensitivity. Transpl Int. 2017;30:295-304.

4. Sharif A, Cohney S. Post-transplantation diabetes - state of the art. Lancet Diabetes Endocrinol. 2016;4:337-49.

5. Ormazabal V, Nair S, Elfeky O, et al. Association between insulin resistance and the development of cardiovascular disease. Cardiovasc Diabetol. 2018;17:122.

6. Oterdoom LH, de Vries AP, Gansevoort RT, et al. Determinants of insulin resistance in renal transplant recipients. Transplantation. 2007;83:29.

7. Valderhaug TG, Hjelmesaeth J, Hartmann A, et al. The association of early post- transplant glucose levels with long-term mortality. Diabetologia. 2011;54:1341-9.

8. Knowler WC, Fowler SE, Hamman RF, et al. 10-Year follow-up of diabetes incidence and weight loss in the Diabetes Prevention Program Outcomes Study. Lancet. 2009;374:1677-86.

9. Li G, Zhang P, Wang J, et al. The long-term effect of lifestyle interventions to prevent diabetes in the China Da Qing Diabetes Prevention Study: a 20-year follow-up study. Lancet. 2008;371:1783-9.

10. Masajtis-Zagajewska A, Muras K, Nowicki M, et al. Effects of a Structured Physical Activity Program on Habitual Physical Activity and Body Composition in Patients With Chronic Kidney Disease and in Kidney Transplant Recipients. Exp Clin Transplant. 2019 Apr;17(2):155-64.

11. O'Hare AM, Towney K, Bacchetti P, et al. Decreased survival among sedentary patients undergoing dialysis: results from the dialysis morbidity and mortality study wave 2. Am J Kidney Dis. 2003;41(2):447-54.

12. Carvalho EV, Reboredo MM, Gomes EP. Physical activity in daily life assessed by an accelerometer in kidney transplant recipients and hemodialysis patients. Transplant Proc. 2014 Jul-Aug; 46 (6) : 17137.

13. Roberts CK, Hevener AL, Barnard RJ. Metabolic syndrome and insulin resistance: underlying causes and modification by exercise training. Compr Physiol. 2013;3:1-58.

14. Kasiske BL, Zeier MG, Chapman JR, et al. KDIGO clinical practice guideline for the care of kidney transplant recipients: A summary. Kidney Int. 2009;9(Suppl 3):1-155.

15. Colberg SR, Sigal RJ, Yardley JE, et al. Physical Activity/Exercise and Diabetes: A Position Statement of the American Diabetes Association. Diabetes Care 2016 Nov; 39 (11): 2065-2079.

16. American College of Sports Medicine. ACSM's Guidelines for Exercise Testing and Prescription. 7th ed. Baltimore: Lippincott Williams \& Wilkins; 2006. 
17. Sanz C, Gautier JF, Hanaire H. Physical exercise for the prevention and treatment of type 2 diabetes. Diabetes Metab. 2010 Nov; 36 (5) : 346-51.

18. Chakkera HA, Weil EJ, Awanson CM, et al. Pretransplant risk score for new-onset diabetes after kidney transplantation. Diabetes Care. 2011;34:2141e5.

19. Sharif A, Moore R, Baboolal K. Influence of lifestyle modification in renal transplant recipients with postprandial hyperglycemia. Transplantation. 2008;85:353-8.

20. Kuningas K, Driscoll J, Mair R, et al. Comparing glycaemic benefits of active versus passive lifestyle intervention in kidney allograft recipients (CAVIAR): a randomised controlled trial. Transplantation. 2020.

21. Klaassen G, Zelle DM, Navis GJ, et al. Lifestyle intervention to improve quality of life and prevent weight gain after renal transplantation: design of the active care after transplantation (ACT) randomized controlled trial. BMC Nephrol. 2017; 181296.

22. Zelle DM, Cerpeleijn E, Stolk RP. Low physical activity and risk of cardiovascular and all-cause mortality in renal transplant recipients. Clin J Am Soc Nephrol. 2011 Apr;6(4):898-905. doi: 10.2215/CJN.03340410. Epub 2011 Mar 3.

23. Byambasukh O, Osté MC, Gomes-Neto AW, et al. Journal of Clinical Medicine. 2020 Feb 3; 9(2): 415.

24. Totti V, Fernhall B, Di Michele R, et al. Medicina. 2020 Apr 16; 56(4): 183.

25. WHO. Global recommendations on physical activity for health. 2010. https://www.who.int/dietphysicalactivity/publications/9789241599979/en/.

26. Jelleyman C, Yates T, O'Donovan G, et al. The effects of high-intensity interval training on glucose regulation and insulin resistance: a meta-analysis. Obes Rev. 2015;16:942-61.

27. Dedinská I, Graňák $K$, Vnučák $M$, et al. Role of sex in post-transplant diabetes mellitus development: are men and women equal? Journal of Diabetes its Complications. 2019;33(4):315-22.

\section{Figures}


A 3

3

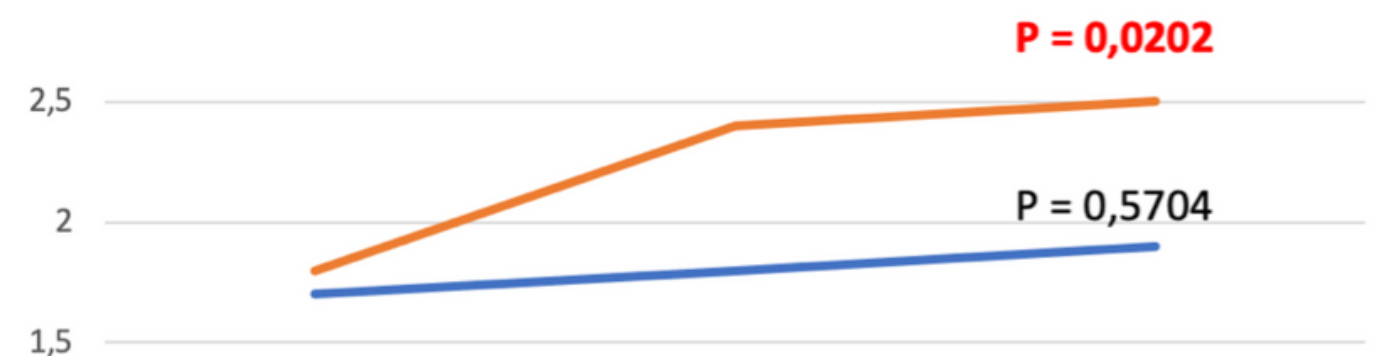

1,5

1

0,5

0 base line $3 \mathrm{M}$ $6 \mathrm{M}$

-monitored group $\longrightarrow$ control group

B

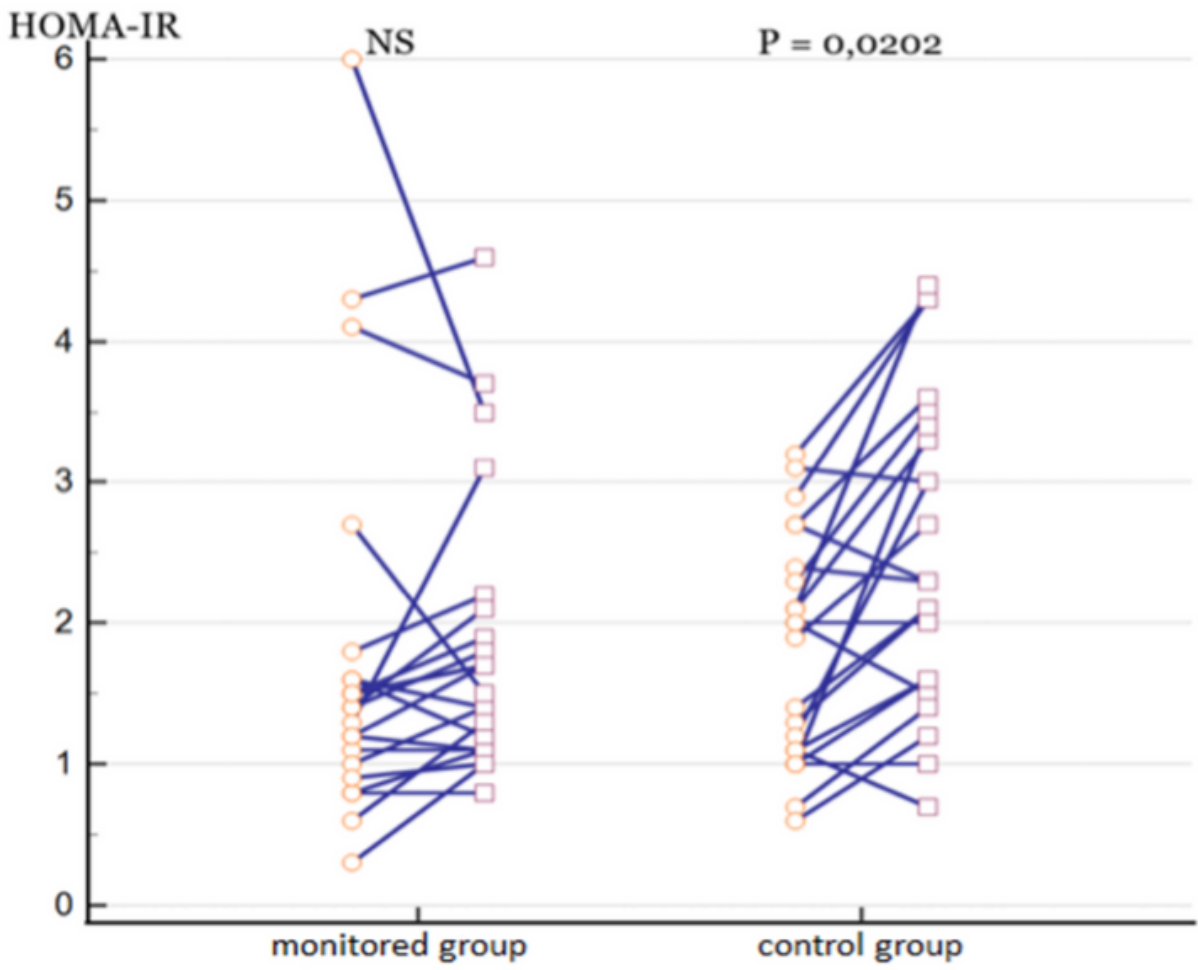

base line

$\square 6 \mathrm{M}$

\section{Figure 1}

Development of HOMA-IR during the study period - A: whole group B: individually 


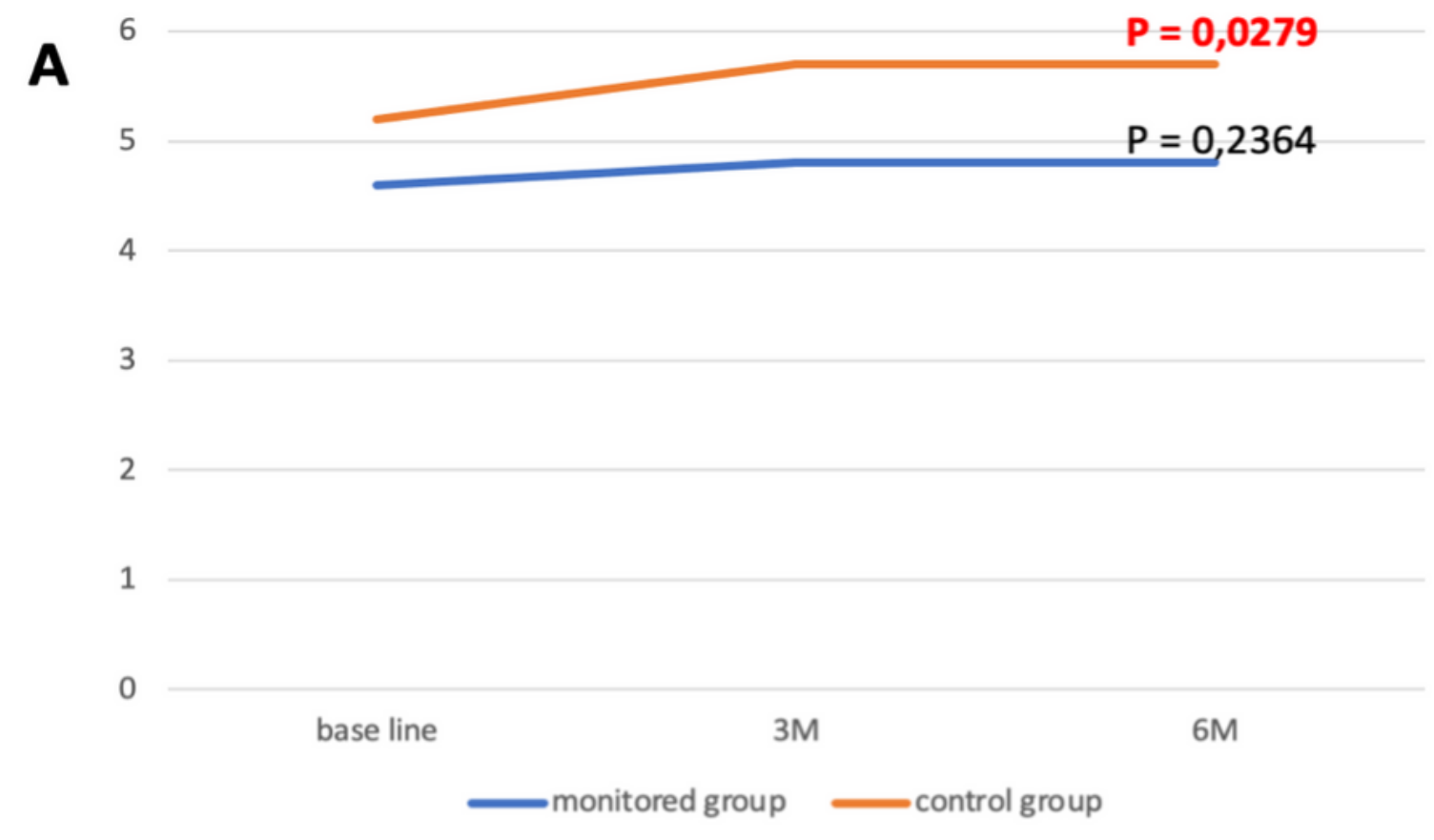

B

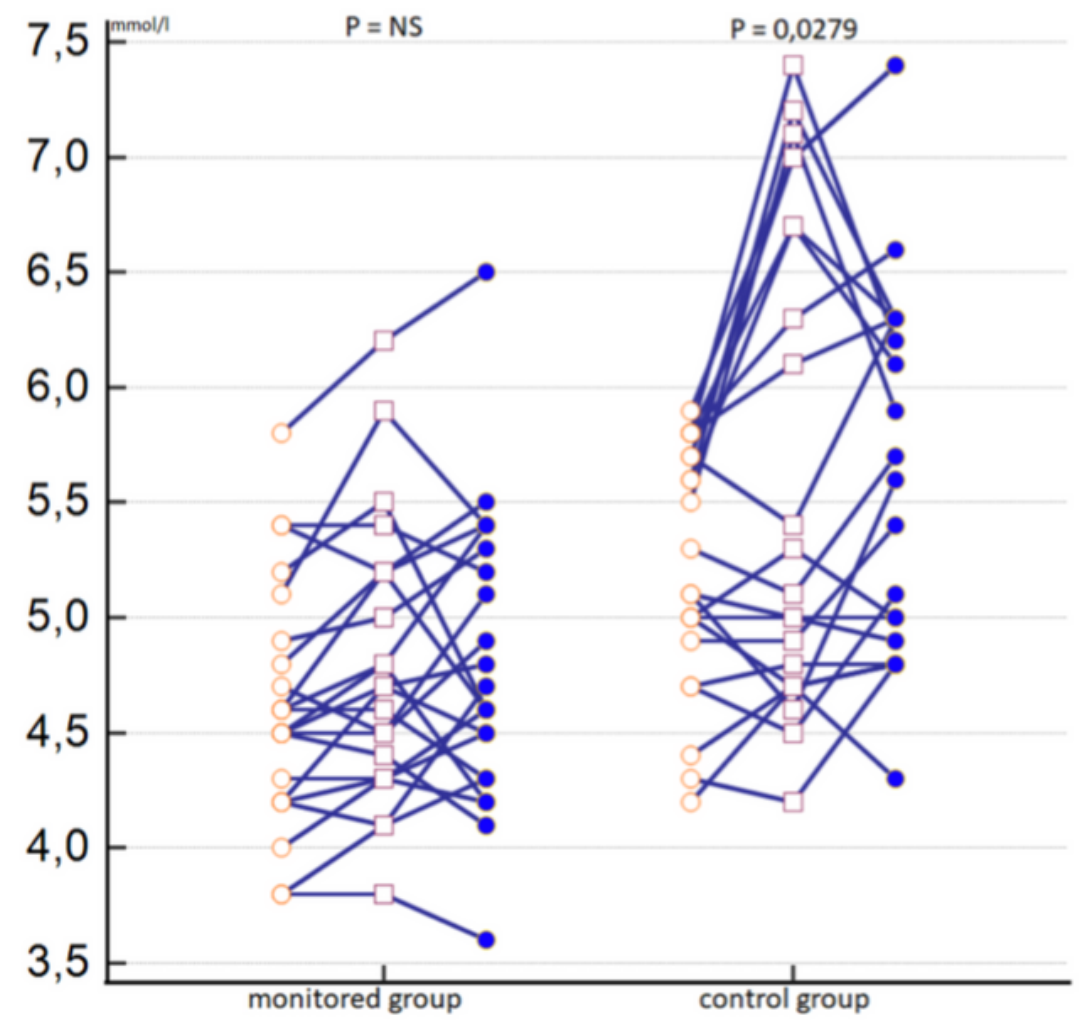

- base line

$\square 3 \mathrm{M}$

- $6 \mathrm{M}$

\section{Figure 2}

Development of fasting glucose during the study period - A: whole group B: individually 


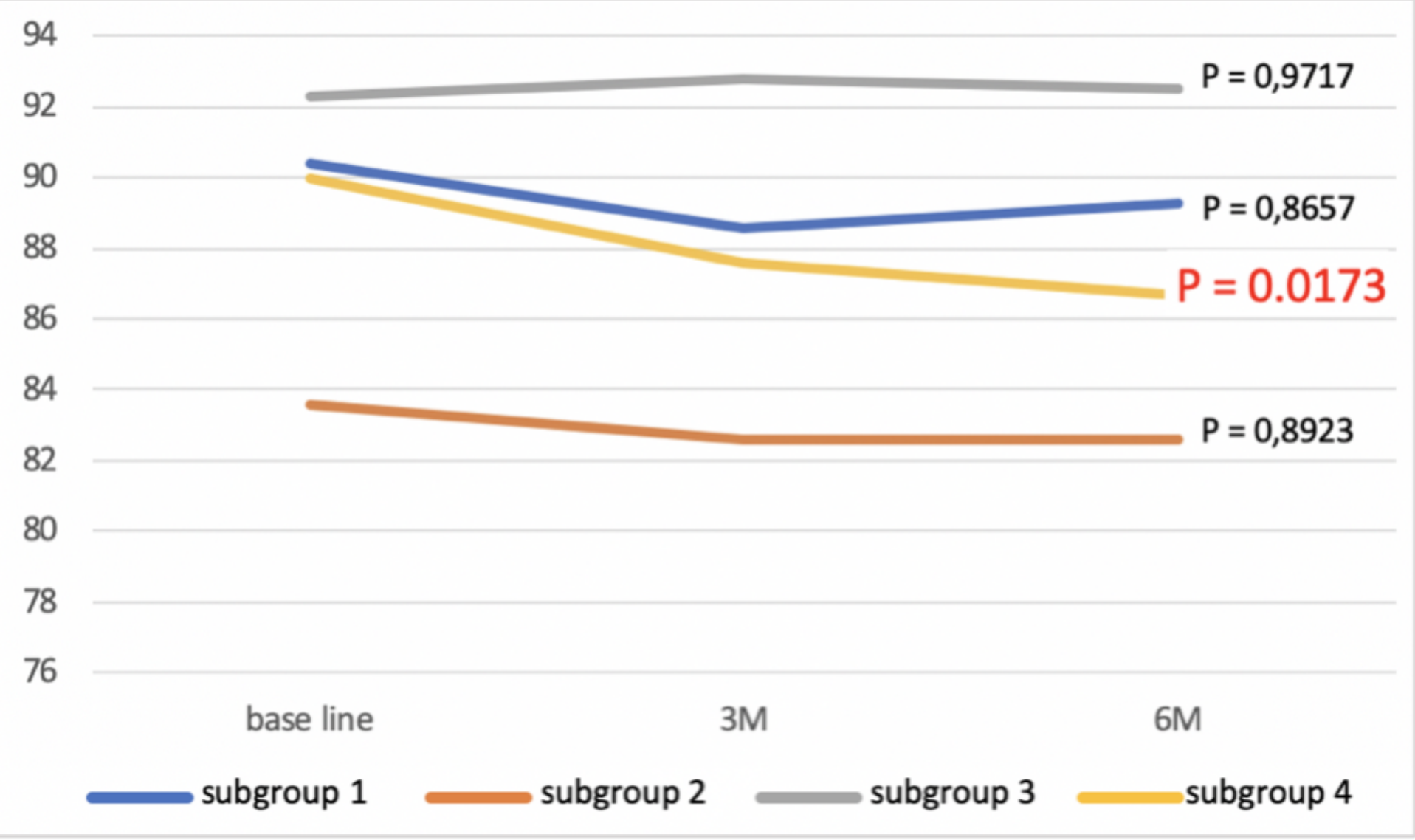

Figure 3

Development of waist circumference in each subgroup during study period (according to activity level).

2,5

2

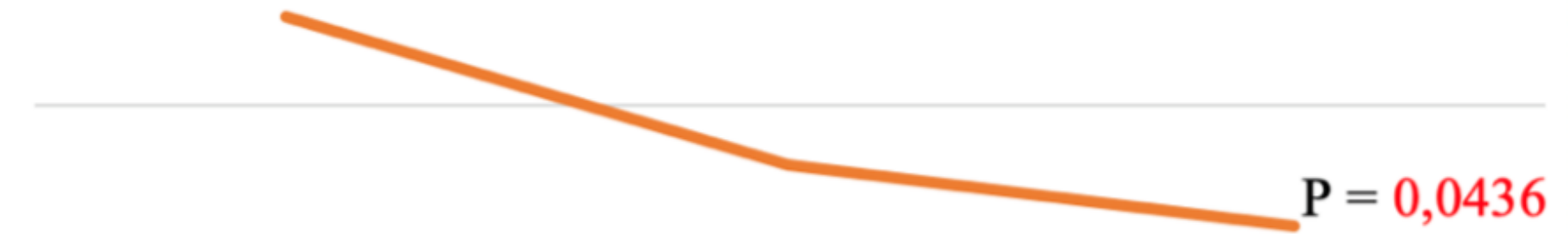

1,5 $\mathrm{P}=0,7605$

1

0,5

0 base line 
Figure 4

Development of triglycerides in each subgroup during study period (according to the type of activity). 